\title{
Fasting Plasma Glucose and Coronary Heart Disease in a Rural Population of North Henan, China
}

\author{
Quan Guo $(\mathbb{D}$, Fei Lin $(\mathbb{D}$, Yi Liu, Yang Li, Xue-Hui Wang, Zhi-Gang Chen, Feng-Hua Lv, \\ Yong-Chun Zhang, Yu Yuan, and Guo-An Zhao
}

Department of Cardiology, First Affiliated Hospital of Xinxiang Medical University, Xinxiang 453100, Henan, China

Correspondence should be addressed to Guo-An Zhao; guoanzhao@xxmu.edu.cn

Received 11 May 2020; Revised 15 September 2020; Accepted 25 September 2020; Published 10 October 2020

Academic Editor: Rana Jamous

Copyright ( $\odot 2020$ Quan Guo et al. This is an open access article distributed under the Creative Commons Attribution License, which permits unrestricted use, distribution, and reproduction in any medium, provided the original work is properly cited.

\begin{abstract}
Even in individuals without diabetes, the incidence of coronary heart disease (CHD) increases with the rise in fasting plasma glucose (FPG); however, the threshold of FPG for CHD in rural areas of China is unclear. We retrospectively examined 2,987 people. Coronary angiography records were used to determine the presence of CHD as well as its severity. Risk factors for CHD and the relationship between different levels of FPG and CHD were analyzed. After adjusting for age, hypertension, dyslipidemia, smoking, drinking, chronic kidney disease, and previous ischemic stroke, the incidence of CHD in nondiabetic women began to increase when FPG exceeded $5.2 \mathrm{mmol} / \mathrm{L}$ (odds ratio $(\mathrm{OR})=1.438,95 \%$ confidence interval $(\mathrm{CI})=1.099-1.880, p=0.008$ ), and the degree of coronary artery lesions also became more severe $(\mathrm{OR}=1.406,95 \% \mathrm{CI}=1.107-1.788, p=0.005)$. However, no such correlations were found in nondiabetic men. In conclusion, among the nondiabetic women in rural areas of northern Henan, both the incidence of CHD and the severity of lesions increased when FPG levels were greater than $5.2 \mathrm{mmol} / \mathrm{L}$, while no significant correlation between FPG and CHD was observed in diabetes-free men.
\end{abstract}

\section{Introduction}

Coronary heart disease (CHD) is a prevalent systemic disease worldwide. It places a heavy economic burden on countries and seriously affects the quality of life. Type 2 diabetes has long been recognized as an independent risk factor for CHD as excessive blood glucose levels can impair endothelial function [1]. However, the pathology of diabetes is a longterm chronic process. Prior to the diagnosis of diabetes, patients would have already developed insulin resistance and blood glucose metabolism disorders, a condition known as prediabetes, which involves impaired fasting glucose (IFG) levels and impaired glucose tolerance [2,3]. The American Diabetes Association (ADA) defines IFG as a fasting plasma glucose (FPG) level of $5.6-6.9 \mathrm{mmol} / \mathrm{L}$, while the World Health Organization (WHO) defines it as an FPG level of 6.1-6.9 mmol/L [4]. Most research studies on prediabetes and cardiovascular disease have been based on these two definitions, but the results have not been uniform [4-7]. The Framingham cohort study demonstrated that there was a positive correlation between the incidence of IFG and cardiovascular events in women, regardless of the definition, but this correlation did not extend to men [8]. A study in Asian populations found an increase in the incidence of cardiovascular events when FPG exceeded $4.9 \mathrm{mmol} / \mathrm{L}$ but did not find any differences between race and sex [9]. Indeed, different regions and races should have their own sets of blood glucose values for defining IFG. A meta-analysis found no increase in CHD risk in subjects without diabetes at baseline when FPG concentrations ranged between $3.9 \mathrm{mmol} / \mathrm{L}$ and $5.6 \mathrm{mmol} / \mathrm{L}$. However, none of the 102 studies published were from China, and the selected populations were from urban areas [10]. Relative to urban populations, rural populations are typically less educated, have poorer health compliance, lack regular medical examinations, have lower sanitation standards, and experience different climates [11]. These differences may account for the discrepancies in their cardiovascular risk factor profiles and the incidence of cardiovascular events [12]. For example, in this research, women in rural areas of northern Henan were found to have 
lower smoking rates than those of the women in the Framingham cohort study. In recent years, the mortality rate of CHD in rural China has exceeded that of the urban population [13]. However, few studies have targeted such population groups. Moreover, the relationship between FPG and coronary atherosclerotic heart diseases in rural areas of northern Henan has not been reported. Therefore, the purpose of this investigation was to retrospectively examine patients in rural areas of northern Henan. We sought to explore the impact of ADA- and WHO-defined IFG on the incidence and severity of CHD and determine whether a more suitable IFG threshold exists for the rural populations of Henan.

\section{Materials and Methods}

2.1. Study Sample. All procedures were approved by the Ethics Committee of the First Affiliated Hospital of Xinxiang Medical College (Approval number 2018118). All data were obtained from the Henan Clinical Data and Sample Research Center for Cardiovascular Diseases database, which was established by the hospital and contains the medical records of all inpatients of the cardiovascular department since January 2016. A total of 3,884 patients from rural areas who underwent coronary angiography between January 2016 and July 2018 were selected from the database. Subsequently, we did not include patients without the necessary indicators for this analysis (417 cases), with previous stent implantation (272 cases), with coronary artery bypass grafting (1 case), with hyperthyroidism (58 cases) or hypothyroidism (14 cases), with severe liver dysfunction (36 cases), with FPG levels less than $3.9 \mathrm{mmol} / \mathrm{L}$ (66 cases), who are pregnant (0 cases), who consumed oral steroidal anti-inflammatory drugs ( 0 cases), and who had malignant tumors (32 cases) or a serious myocardial bridge ( 1 case) in the final analysis. A total of 2,987 patients were eventually included, and all participants had complete information, including name, hospital number, personal code, age, sex, FPG, liver function, kidney function, blood pressure, smoking history, drinking history, past medical history, blood lipids (including total cholesterol, triglycerides, high-density lipoprotein cholesterol, and low-density lipoprotein cholesterol), coronary angiography records, and Gensini scores (GS). There were no demographic differences between the excluded data and the final analyzed data.

2.2. Baseline and Definition. All patients underwent coronary angiography at the Heart Center of the First Affiliated Hospital of Xinxiang Medical College between January 2016 and July 2018. Two or more experienced physicians assessed the angiographic results. CHD was defined as stenosis of $\geq 50 \%$ in at least one major or branch vessel. The degree of coronary artery lesions was divided into 4 groups according to the GS [14]: normal $(\mathrm{GS}<1)$, mild lesions $(1 \leq \mathrm{GS}<20)$, moderate lesions $(20 \leq \mathrm{GS}<49)$, and severe lesions ( $\mathrm{GS} \geq 49)$.

All patients underwent a venous blood draw for glucose measurement after fasting for more than 8 hours. Those with
FPG $\geq 7.0 \mathrm{mmol} / \mathrm{L}$, a history of diabetes, or currently using hypoglycemic drugs (including oral hypoglycemic agents and insulin) were classified as having diabetes. IFG was divided into IFG-ADA (5.6-6.9 mmol/L), IFG-WHO (6.1-6.9 mmol/L) [4], or other specific cut-off points, according to the different definitions.

Hypertension was defined as a history of hypertension, the current use of oral antihypertensive drugs, or a systolic blood pressure $\geq 140 \mathrm{mmHg}$ or diastolic blood pressure $\geq 90 \mathrm{mmHg}$ at admission.

Smoking was defined as current or previous smoking habits involving an average of more than one cigarette per day for more than 6 months.

Drinking was defined as a current or past history of alcohol consumption, with an average daily amount $\geq 40 \mathrm{~g}$ for a period longer than 6 months.

Dyslipidemia was considered in patients with triglycerides $\geq 2.27 \mathrm{mmol} / \mathrm{L}$, total cholesterol $\geq 6.19 \mathrm{mmol} / \mathrm{L}$, lowdensity lipoprotein cholesterol $\geq 4.14 \mathrm{mmol} / \mathrm{L}$, and/or highdensity lipoprotein cholesterol $<1.04 \mathrm{mmol} / \mathrm{L}$.

Patients with a history of chronic kidney disease or men and women with serum creatinine levels $>105 \mu \mathrm{mol} /$ $\mathrm{L}$ and $>100 \mu \mathrm{mol} / \mathrm{L}$, respectively, were classified as having chronic kidney disease.

Ischemic stroke was considered based on a history of ischemic stroke, including neurological deficits and/or imaging evidence.

2.3. Statistical Analyses. The incidence of CHD was first assessed using a univariate analysis of diabetes and nondiabetes patients in all included populations. Binary logistic regression was then used to establish a multivariate model for the analysis of CHD risk factors. Then, all patients with diabetes were excluded, and blood glucose was grouped according to the ADA, WHO, or other cut-off criteria. Univariate analysis of CHD incidence and baseline data were assessed between the groups. To eliminate the effects of confounding factors, $1: 1$ propensity score matching (PSM) was applied to eliminate the differences between the IFG and normal groups and to calculate for the adjusted odds ratios (OR). The covariates included in the PSM model were age, hypertension, smoking, drinking, dyslipidemia, chronic kidney disease, and previous ischemic stroke. After PSM, each variable was compared to ensure comparability between both groups. Multivariate binary logistic regression was used to explore the relationship between FPG and CHD in different populations. In all univariate analyses of the incidence of $\mathrm{CHD}$, count data were assessed using the chisquared test. Normally distributed continuous data were expressed are mean \pm variance and were compared using the selected independent Student's $t$-test, while nonnormally distributed data are expressed as median and interquartile range and were evaluated using the Mann-Whitney $U$ test. Multivariate ordered logistic regression was used to analyze the relationship between IFG and the severity of coronary lesions. Continuous variables were used when analyzing the relationship between CHD and FPG in the different populations. The factors included in all multivariate models were 
baseline data with statistical differences in the univariate analysis. All statistical analyses, including PSM, were performed with SPSS software 25.0 (IBM, USA). $p$ values were 2 -tailed, and $p<0.05$ was considered significant.

\section{Results}

A total of 2,987 patients were enrolled in the study, including $53.8 \%$ men $(n=1,607), 66.9 \%$ with CHD $(n=1,997), 22.3 \%$ with diabetes $(n=665), 20 \%$ with IFG-ADA $(n=598)$, and $8.7 \%$ with IFG-WHO $(n=260)$.

3.1. Effects of Diabetes on CHD. In all populations, univariate analysis showed an increased incidence of CHD in patients with diabetes, with an OR of 2.754 (95\% CI = 2.221-3.416, $p<0.01)$. After adjusting for age, sex, hypertension, dyslipidemia, smoking, alcohol consumption, and ischemic stroke in the multivariate model, an adjusted OR of 2.593 $(95 \% \mathrm{CI}=2.067-3.253, p<0.01)$ was found (Tables 1 and 2 ).

Given the higher risk ratio of men to women concerning the incidence of CHD, the adjusted OR was $2.153(95 \%$ $\mathrm{CI}=1.717-2.700, p<0.01)$, and the sexes were analyzed separately.

Consistent with the general population, the incidence of CHD was elevated in both diabetic male and female patients. In men, univariate analysis showed that the OR of $\mathrm{CHD}$ in patients with diabetes was $3.003 \quad(95 \%$ $\mathrm{CI}=2.095-4.303, \quad p<0.01)$. After correcting for age, smoking, hypertension, dyslipidemia, and ischemic stroke history, the adjusted OR was $2.774(95 \% \mathrm{CI}=1.913-4.020$, $p<0.01)$. In women, the OR was $3.069 \quad(95 \%$ $\mathrm{CI}=2.327-4.047, p<0.01)$, and the adjusted $\mathrm{OR}$ was 2.496 (95\% CI $=1.868-3.335, p<0.01)$. In the multivariate model, hypertension was a risk factor for CHD in women but not in men. In contrast, smoking was a risk factor for CHD in men but not in women. In fact, the smoking rate of women with CHD was very low (1.11\%).

3.2. Impact of IFG on CHD. To explore the impact of IFG on morbidity, we excluded patients with diabetes, and the remaining population was divided into groups according to the diagnostic criteria of ADA and WHO, namely, an ADAdefined normal group and IFG group or a WHO-defined normal group and IFG group. They were analyzed separately by sex.

In men, univariate analysis showed an increased incidence of CHD in the WHO-defined IFG group, but the result was not significant $(76.6 \%$ versus $69.9 \%, p=0.115)$. The incidence of CHD in the ADA-defined IFG group was also not significant relative to the normal group $(70.5 \%$ versus $70.7 \%, p=0.944)$. Owing to the decreased sample size in men with FPG greater than $6.1 \mathrm{mmol} / \mathrm{L}$, the degree of difference may have been affected. New cut-off points between $5.6 \mathrm{mmol} / \mathrm{L}$ and $6.1 \mathrm{mmol} / \mathrm{L}$ at $0.1 \mathrm{mmol} / \mathrm{L}$ increments were set to avoid missing potentially important cut-off thresholds. Univariate analysis showed that when the cut-off points were $5.8 \mathrm{mmol} / \mathrm{L}, 5.9 \mathrm{mmol} / \mathrm{L}$, and $6.0 \mathrm{mmol} / \mathrm{L}$, there were significant differences in the incidence of CHD between the IFG and normal blood glucose groups. After using PSM to rule out confounding factors, the IFG group, without any cut-off points, was justified. Although no differences in baseline data were observed before PSM when using cut-off points $5.6 \mathrm{mmol} / \mathrm{L}$ to $5.9 \mathrm{mmol} / \mathrm{L}$, PSM was still used to further reduce the difference in baseline data between the two groups (Table 3 and Figure 1).

In women, both the ADA- and WHO-defined IFG groups showed significant increases in $\mathrm{CHD}$ incidence during univariate analysis. To better eliminate the differences in baseline data, PSM was applied. After adjusting for the confounding factors, significantly increased CHD incidence still remained. To identify whether the difference in the incidence rate already existed at lower FPG levels, a plurality of cut-off points at $5.6 \mathrm{mmol} / \mathrm{L}$ or less at intervals of $0.1 \mathrm{mmol} / \mathrm{L}$ were set, and PSM was separately performed. There were significant differences in the incidence of CHD between the IFG and normal groups when a $5.2 \mathrm{mmol} / \mathrm{L}$ cut-off point was selected $(\mathrm{OR}=1.438,95 \% \mathrm{CI}=1.099-1.880, p=0.008)$. At cut-off points $5.0 \mathrm{mmol} / \mathrm{L}$ and $5.1 \mathrm{mmol} / \mathrm{L}$, although $\mathrm{CHD}$ was higher in the IFG group than in the normal group, it was not significant (Table 4 and Figure 2). The ordered logistic regression analysis revealed that $\mathrm{CHD}$ lesions were more severe in the IFG group when the cut-off point was set at $5.2 \mathrm{mmol} / \mathrm{L}(\mathrm{OR}=1.406,95 \% \mathrm{CI}=1.107-1.788$, $p=0.005$, Table 5).

3.3. Additional Analysis. To determine the reason why elevated fasting glucose only affected the incidence of CHD in women but not in men, we compared the baseline characteristics of men and women with angiographically confirmed CHD. Relative to female CHD patients, male $\mathrm{CHD}$ patients had lower FPG levels, age at onset, and incidence of hypertension but significantly higher dyslipidemia, smoking, and drinking habits. To explore whether the differences between men and women were caused by the different proportions of risk factors, we established 5 multivariate models according to smoking status, drinking habits, dyslipidemia, age ( $\geq 60$ years old), and hypertension. The effects of FPG on the incidence of CHD occurred in the group without dyslipidemia (adjusted $\mathrm{OR}=1.252, \quad 95 \%$ $\mathrm{CI}=1.042-1.504$ ) and in nonsmokers (adjusted $\mathrm{OR}=1.234$, $95 \% \mathrm{CI}=1.038-1.468)$. This was in line with the higher proportions of hyperlipidemia and smokers in men (Tables 6 and 7).

\section{Discussion}

4.1. Fasting Glucose and CHD. In the present investigation, we found that elevated levels of FPG cause an increase in $\mathrm{CHD}$ incidence in women from rural areas of northern Henan, China, but not in men. These results are similar to those of the Framingham cohort study [8]. However, a novel finding of our study was that diabetes-free women were associated with a significantly increased incidence of $\mathrm{CHD}$ 
TABLE 1: Baseline characteristics of patients with and without CHD.

\begin{tabular}{|c|c|c|c|c|c|c|c|c|c|}
\hline & \multicolumn{2}{|c|}{ All participants } & \multirow[b]{2}{*}{$\begin{array}{c}p \\
\text { value }\end{array}$} & \multicolumn{2}{|c|}{ Male } & \multirow[b]{2}{*}{$\begin{array}{c}p \\
\text { value }\end{array}$} & \multicolumn{2}{|c|}{ Female } & \multirow[b]{2}{*}{$\begin{array}{c}p \\
\text { value }\end{array}$} \\
\hline & $\begin{array}{c}\text { CHD } \\
(n=1997)\end{array}$ & $\begin{array}{l}\text { No CHD } \\
(n=990)\end{array}$ & & $\begin{array}{c}\text { CHD } \\
(n=1187)\end{array}$ & $\begin{array}{l}\text { No CHD } \\
(n=420)\end{array}$ & & $\begin{array}{c}\text { CHD } \\
(n=810)\end{array}$ & $\begin{array}{l}\text { No CHD } \\
(n=570)\end{array}$ & \\
\hline Diabetes & $546(27.3 \%)$ & $119(12.0 \%)$ & $<0.001$ & $273(20.0 \%)$ & $38(9.0 \%)$ & $<0.001$ & $273(29.3 \%)$ & $81(14.2 \%)$ & $<0.001$ \\
\hline Age & $61(53-67)$ & $54(48-62)$ & $<0.001$ & $59(51-66)$ & $52(46-60)$ & $<0.001$ & $62 \pm 9$ & $57 \pm 9$ & $<0.001$ \\
\hline Male & $1187(59.4 \%)$ & $420(42.4 \%)$ & $<0.001$ & / & l & l & l & I & I \\
\hline Smoking & $793(39.7 \%)$ & $257(26.0 \%)$ & $<0.001$ & $784(66.0 \%)$ & $252(60.0 \%)$ & 0.026 & $9(1.1 \%)$ & $5(0.9 \%)$ & 0.669 \\
\hline Drinking & $404(20.2 \%)$ & $147(14.8 \%)$ & $<0.001$ & $398(33.5 \%)$ & $143(34.0 \%)$ & 0.847 & $6(0.7 \%)$ & $4(0.7 \%)$ & 0.933 \\
\hline Hypertension & $1442(72.2 \%)$ & $623(62.9 \%)$ & $<0.001$ & $796(67.1 \%)$ & $254(60.5 \%)$ & 0.015 & $646(79.8 \%)$ & $369(64.7 \%)$ & $<0.001$ \\
\hline Dyslipidemia & $1029(51.5 \%)$ & $373(37.7 \%)$ & $<0.001$ & $668(56.3 \%)$ & $187(44.5 \%)$ & $<0.001$ & $361(44.6 \%)$ & $186(32.6 \%)$ & $<0.001$ \\
\hline $\begin{array}{l}\text { Previous ischemic } \\
\text { stroke }\end{array}$ & $263(13.2 \%)$ & $70(7.1 \%)$ & $<0.001$ & $160(13.5 \%)$ & $23(5.5 \%)$ & $<0.001$ & $103(12.7 \%)$ & $47(8.2 \%)$ & 0.009 \\
\hline $\begin{array}{l}\text { Chronic kidney } \\
\text { disease }\end{array}$ & $20(1.0 \%)$ & $8(0.8 \%)$ & 0.606 & $15(1.3 \%)$ & $4(1.0 \%)$ & 0.612 & $5(0.6 \%)$ & $4(0.7 \%)$ & 0.848 \\
\hline
\end{tabular}

CHD: coronary heart disease.

TABle 2: Multivariate analysis of risk factors for coronary heart disease.

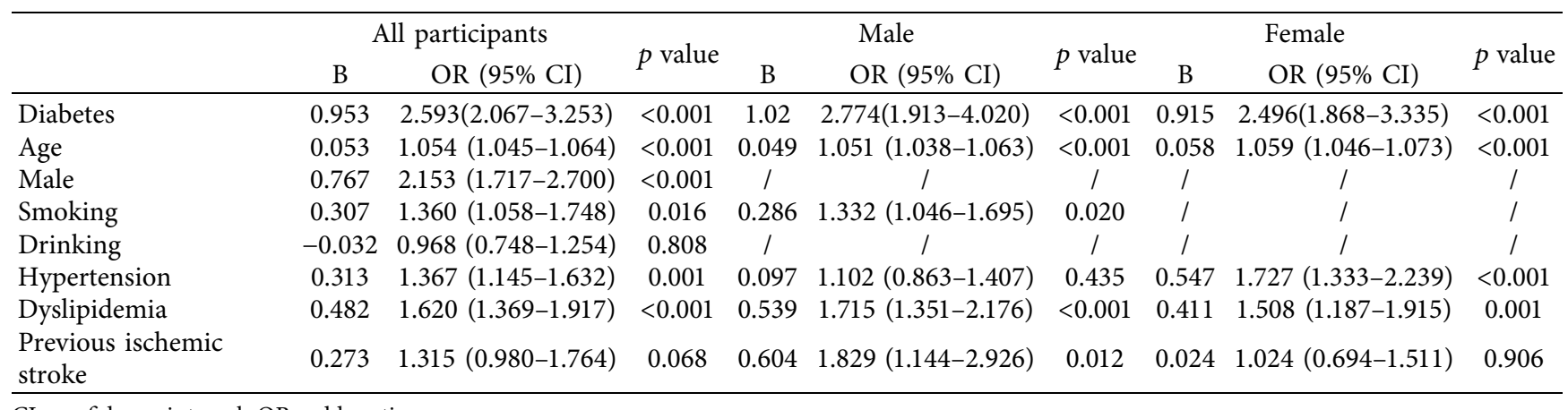

CI: confidence interval; OR: odds ratio.

TABLE 3: Baseline characteristics of IFG and normal groups defined by different cut-off points before and after PSM in male patients.

\begin{tabular}{|c|c|c|c|c|c|c|c|c|c|c|}
\hline \multicolumn{3}{|c|}{ Cut-off point of IFG } & Age & Hypertension & Smoking & Drinking & Dyslipidemia & $\begin{array}{c}\text { Chronic } \\
\text { kidney } \\
\text { disease }\end{array}$ & $\begin{array}{c}\text { Previous } \\
\text { ischemic } \\
\text { stroke }\end{array}$ & $\mathrm{CHD}$ \\
\hline \multirow{5}{*}{$5.6 \mathrm{mmol} / \mathrm{L}$} & \multirow{3}{*}{$\begin{array}{l}\text { Before } \\
\text { PSM }\end{array}$} & $\begin{array}{c}\mathrm{NG} \\
(n=989)\end{array}$ & $\begin{array}{c}57 \\
(49-64)\end{array}$ & $622(62.9 \%)$ & $\begin{array}{c}646 \\
(65.3 \%)\end{array}$ & $\begin{array}{c}341 \\
(34.5 \%)\end{array}$ & $487(49.2 \%)$ & $11(1.1 \%)$ & $108(10.9 \%)$ & $\begin{array}{c}6997 \\
(70.5 \%)\end{array}$ \\
\hline & & $\begin{array}{c}\text { IFG } \\
(n=307)\end{array}$ & $\begin{array}{c}57 \\
(50-64)\end{array}$ & $200(65.1 \%)$ & $\begin{array}{c}200 \\
(65.1 \%)\end{array}$ & $\begin{array}{c}96 \\
(31.3 \%)\end{array}$ & $163(53.1 \%)$ & $7(2.3 \%)$ & $24(7.8 \%)$ & $\begin{array}{c}217 \\
(70.7 \%)\end{array}$ \\
\hline & & $p$ value & 0.469 & 0.474 & 0.956 & 0.299 & 0.238 & 0.159 & 0.116 & 0.944 \\
\hline & \multirow{3}{*}{$\begin{array}{l}\text { After } \\
\text { PSM }\end{array}$} & $\begin{array}{c}\text { NG } \\
(n=306)\end{array}$ & $57 \pm 11$ & $188(61.4 \%)$ & $\begin{array}{c}198 \\
(64.7 \%)\end{array}$ & $\begin{array}{c}80 \\
(26.1 \%)\end{array}$ & $151(49.3 \%)$ & $4(1.3 \%)$ & $38(12.4 \%)$ & $\begin{array}{c}231 \\
(75.5 \%)\end{array}$ \\
\hline & & $\begin{array}{c}\text { IFG } \\
(n=306)\end{array}$ & $57 \pm 10$ & $199(65 \%)$ & $\begin{array}{c}200 \\
(65.4 \%)\end{array}$ & $\begin{array}{c}96 \\
(31.4 \%)\end{array}$ & $162(52.9 \%)$ & $7(2.3 \%)$ & $24(7.8 \%)$ & $\begin{array}{c}216 \\
(70.6 \%)\end{array}$ \\
\hline \multirow{7}{*}{$5.7 \mathrm{mmol} / \mathrm{L}$} & & $p$ value & 0.703 & 0.356 & 0.865 & 0.153 & 0.374 & 0.361 & 0.061 & 0.172 \\
\hline & \multirow{3}{*}{$\begin{array}{c}\text { Before } \\
\text { PSM }\end{array}$} & $\begin{array}{c}\text { NG } \\
(n=1031)\end{array}$ & $\begin{array}{c}57 \\
(49-64)\end{array}$ & $648(62.9 \%)$ & $\begin{array}{c}671 \\
(65.1 \%)\end{array}$ & $\begin{array}{c}350 \\
(33.9 \%)\end{array}$ & $512(49.7 \%)$ & $12(1.2 \%)$ & $111(10.8 \%)$ & $\begin{array}{c}722 \\
(70 \%)\end{array}$ \\
\hline & & $\begin{array}{c}\text { IFG } \\
(n=265)\end{array}$ & $\begin{array}{c}56 \\
(50-64)\end{array}$ & $174(65.7 \%)$ & $\begin{array}{c}175 \\
(66 \%)\end{array}$ & $\begin{array}{c}87 \\
(32.8 \%)\end{array}$ & $138(52.1 \%)$ & $6(2.3 \%)$ & $21(7.9 \%)$ & $\begin{array}{c}192 \\
(72.5 \%)\end{array}$ \\
\hline & & $p$ value & 0.748 & 0.397 & 0.771 & 0.731 & 0.483 & 0.233 & 0.173 & 0.440 \\
\hline & \multirow{3}{*}{$\begin{array}{l}\text { After } \\
\text { PSM }\end{array}$} & $\begin{array}{c}\text { NG } \\
(n=265)\end{array}$ & $58 \pm 11$ & $158(59.6 \%)$ & $\begin{array}{c}176 \\
(66.4 \%)\end{array}$ & $\begin{array}{c}80 \\
(30.2 \%)\end{array}$ & $116(43.8 \%)$ & $2(0.8 \%)$ & $33(12.5 \%)$ & $\begin{array}{c}196 \\
(74 \%)\end{array}$ \\
\hline & & $\begin{array}{c}\text { IFG } \\
(n=265)\end{array}$ & $57 \pm 10$ & $174(65.7 \%)$ & $\begin{array}{c}175 \\
(66 \%)\end{array}$ & $\begin{array}{c}87 \\
(32.8 \%)\end{array}$ & $138(52.1 \%)$ & $6(2.3 \%)$ & $21(7.9 \%)$ & $\begin{array}{c}192 \\
(72.5 \%)\end{array}$ \\
\hline & & $p$ value & 0.493 & 0.151 & 0.927 & 0.513 & 0.056 & 0.285 & 0.085 & 0.695 \\
\hline
\end{tabular}


TABLE 3: Continued.

\begin{tabular}{|c|c|c|c|c|c|c|c|c|c|c|}
\hline \multicolumn{3}{|c|}{ Cut-off point of IFG } & Age & Hypertension & Smoking & Drinking & Dyslipidemia & $\begin{array}{c}\text { Chronic } \\
\text { kidney } \\
\text { disease }\end{array}$ & $\begin{array}{c}\text { Previous } \\
\text { ischemic } \\
\text { stroke }\end{array}$ & CHD \\
\hline \multirow{5}{*}{$5.8 \mathrm{mmol} / \mathrm{L}$} & \multirow{3}{*}{$\begin{array}{c}\text { Before } \\
\text { PSM }\end{array}$} & $\begin{array}{c}\mathrm{NG} \\
(n=1081)\end{array}$ & $\begin{array}{c}57 \\
(49-64)\end{array}$ & $679(62.8 \%)$ & $\begin{array}{c}700 \\
(64.8 \%)\end{array}$ & $\begin{array}{c}365 \\
(33.8 \%)\end{array}$ & $538(49.8 \%)$ & $13(1.2 \%)$ & $112(10.4 \%)$ & $\begin{array}{c}747 \\
(69.1 \%)\end{array}$ \\
\hline & & $\begin{array}{c}\text { IFG } \\
(n=215)\end{array}$ & $\begin{array}{c}58 \\
(51-65)\end{array}$ & $143(66.5 \%)$ & $\begin{array}{c}146 \\
(67.9 \%)\end{array}$ & $\begin{array}{c}72 \\
(33.5 \%)\end{array}$ & $112(52.1 \%)$ & $5(2.3 \%)$ & $20(9.3 \%)$ & $\begin{array}{c}167 \\
(77.7 \%)\end{array}$ \\
\hline & & $p$ value & 0.096 & 0.304 & 0.375 & 0.938 & 0.534 & 0.202 & 0.639 & 0.012 \\
\hline & \multirow{3}{*}{$\begin{array}{l}\text { After } \\
\text { PSM }\end{array}$} & $\begin{array}{c}\text { NG } \\
(n=204)\end{array}$ & $57 \pm 9$ & $136(66.7 \%)$ & $\begin{array}{c}142 \\
(69.6 \%)\end{array}$ & $\begin{array}{c}65 \\
(31.9 \%)\end{array}$ & $108(52.9 \%)$ & $1(0.5 \%)$ & $20(9.8 \%)$ & $\begin{array}{c}152 \\
(74.5 \%)\end{array}$ \\
\hline & & $\begin{array}{c}\text { IFG } \\
(n=204)\end{array}$ & $57 \pm 10$ & $136(66.7 \%)$ & $\begin{array}{c}141 \\
(69.1 \%)\end{array}$ & $\begin{array}{c}70 \\
(34.3 \%)\end{array}$ & $107(52.5 \%)$ & $5(2.5 \%)$ & $19(9.3 \%)$ & $\begin{array}{c}158 \\
(77.5 \%)\end{array}$ \\
\hline \multirow{7}{*}{$5.9 \mathrm{mmol} / \mathrm{L}$} & & $p$ value & 0.987 & 1 & 0.914 & 0.599 & 0.921 & 0.215 & 0.866 & 0.487 \\
\hline & \multirow{3}{*}{$\begin{array}{c}\text { Before } \\
\text { PSM }\end{array}$} & $\begin{array}{c}\text { NG } \\
(n=1109)\end{array}$ & $\begin{array}{c}57 \\
(49-64)\end{array}$ & $693(62.5 \%)$ & $\begin{array}{c}713 \\
(64.3 \%)\end{array}$ & $\begin{array}{c}371 \\
(33.5 \%)\end{array}$ & $555(50 \%)$ & $14(1.3 \%)$ & $113(10.2 \%)$ & $\begin{array}{c}769 \\
(69.3 \%)\end{array}$ \\
\hline & & $\begin{array}{c}\text { IFG } \\
(n=187)\end{array}$ & $\begin{array}{c}58 \\
(51-65)\end{array}$ & $129(69 \%)$ & $\begin{array}{c}133 \\
(71.1 \%)\end{array}$ & $\begin{array}{c}66 \\
(35.3 \%)\end{array}$ & $95(50.8 \%)$ & $4(2.1 \%)$ & $19(10.2 \%)$ & $\begin{array}{c}145 \\
(77.5 \%)\end{array}$ \\
\hline & & $p$ value & 0.069 & 0.088 & 0.070 & 0.622 & 0.848 & 0.314 & 0.990 & 0.023 \\
\hline & \multirow{3}{*}{$\begin{array}{l}\text { After } \\
\text { PSM }\end{array}$} & $\begin{array}{c}\text { Normal } \\
(n=166)\end{array}$ & $57 \pm 9$ & $116(69.9 \%)$ & $\begin{array}{c}120 \\
(72.3 \%)\end{array}$ & $\begin{array}{c}53 \\
(31.9 \%)\end{array}$ & $90(54.2 \%)$ & $0(0 \%)$ & $11(6.6 \%)$ & $\begin{array}{c}120 \\
(72.3 \%)\end{array}$ \\
\hline & & $\begin{array}{c}\text { IFG } \\
(n=166)\end{array}$ & $57 \pm 9$ & $116(69.9 \%)$ & $\begin{array}{c}120 \\
(72.3 \%)\end{array}$ & $\begin{array}{c}60 \\
(36.1 \%)\end{array}$ & $90(54.2 \%)$ & $3(1.8 \%)$ & $11(6.6 \%)$ & $\begin{array}{c}126 \\
(75.9 \%)\end{array}$ \\
\hline & & $p$ value & 1 & 1 & 0.720 & 0.417 & 1 & 0.248 & 1 & 0.452 \\
\hline \multirow{5}{*}{$6 \mathrm{mmol} / \mathrm{L}$} & \multirow{3}{*}{$\begin{array}{c}\text { Before } \\
\text { PSM }\end{array}$} & $\begin{array}{c}\text { NG } \\
(n=1144)\end{array}$ & $\begin{array}{c}57 \\
(49-64)\end{array}$ & $716(62.6 \%)$ & $\begin{array}{c}738 \\
(64.5 \%)\end{array}$ & $\begin{array}{c}380 \\
(33.2 \%)\end{array}$ & $571(49.9 \%)$ & $15(1.3 \%)$ & $118(10.3 \%)$ & $\begin{array}{c}795 \\
(69.5 \%)\end{array}$ \\
\hline & & $\begin{array}{c}\text { IFG } \\
(n=152)\end{array}$ & $\begin{array}{c}60 \\
(51-66)\end{array}$ & $106(69.7 \%)$ & $\begin{array}{c}108 \\
(71.1 \%)\end{array}$ & $\begin{array}{c}57 \\
(37.5 \%)\end{array}$ & $79(52 \%)$ & $3(2.0 \%)$ & $14(9.2 \%)$ & $\begin{array}{c}119 \\
(78.3 \%)\end{array}$ \\
\hline & & $p$ value & 0.013 & 0.086 & 0.111 & 0.294 & 0.633 & 0.459 & 0.672 & 0.025 \\
\hline & \multirow{3}{*}{$\begin{array}{l}\text { After } \\
\text { PSM }\end{array}$} & $\begin{array}{c}\text { NG } \\
(n=152)\end{array}$ & $60 \pm 9$ & $98(64.5 \%)$ & $\begin{array}{c}106 \\
(69.7 \%)\end{array}$ & $\begin{array}{c}45 \\
(29.6 \%)\end{array}$ & $65(42.8 \%)$ & $0(0 \%)$ & $23(15.1 \%)$ & $\begin{array}{c}124 \\
(81.6 \%)\end{array}$ \\
\hline & & $\begin{array}{c}\text { IFG } \\
(n=152)\end{array}$ & $59 \pm 10$ & $106(69.7 \%)$ & $\begin{array}{c}108 \\
(71.1 \%)\end{array}$ & $\begin{array}{c}57 \\
(37.5 \%)\end{array}$ & $79(52 \%)$ & $3(2 \%)$ & $14(9.2 \%)$ & $\begin{array}{c}119 \\
(78.3 \%)\end{array}$ \\
\hline \multirow{7}{*}{$6.1 \mathrm{mmol} / \mathrm{L}$} & & $p$ value & 0.100 & 0 & 0.802 & 0.145 & 0.108 & 0.248 & 0.114 & 0.474 \\
\hline & \multirow{3}{*}{$\begin{array}{c}\text { Before } \\
\text { PSM }\end{array}$} & $\begin{array}{c}\text { NG } \\
(n=1168)\end{array}$ & $\begin{array}{c}57 \\
(49-64)\end{array}$ & $730(62.5 \%)$ & $\begin{array}{c}754 \\
(64.6 \%)\end{array}$ & $\begin{array}{c}388 \\
(33.2 \%)\end{array}$ & 583 (49.9\%) & $16(1.4 \%)$ & 119 (10.2\%) & $\begin{array}{c}816 \\
(69.9 \%)\end{array}$ \\
\hline & & $\begin{array}{c}\text { IFG } \\
(n=128)\end{array}$ & $\begin{array}{c}61 \\
(51-66)\end{array}$ & $92(71.9 \%)$ & $\begin{array}{c}92 \\
(71.9 \%)\end{array}$ & $\begin{array}{c}49 \\
(38.3 \%)\end{array}$ & $67(52.3 \%)$ & $2(1.6 \%)$ & $13(10.2 \%)$ & $\begin{array}{c}98 \\
(76.6 \%)\end{array}$ \\
\hline & & $p$ value & 0.023 & 0.037 & 0.099 & 0.250 & 0.602 & 0.696 & 0.991 & 0.115 \\
\hline & \multirow{3}{*}{$\begin{array}{l}\text { After } \\
\text { PSM }\end{array}$} & $\begin{array}{c}\text { NG } \\
(n=128)\end{array}$ & $\begin{array}{c}60 \\
(51-67)\end{array}$ & $92(71.9 \%)$ & $\begin{array}{c}83 \\
(64.8 \%)\end{array}$ & $\begin{array}{c}37 \\
(28.9 \%)\end{array}$ & $57(44.5 \%)$ & $1(0.8 \%)$ & $23(18 \%)$ & $\begin{array}{c}101 \\
(78.9 \%)\end{array}$ \\
\hline & & $\begin{array}{c}\text { IFG } \\
(n=128)\end{array}$ & $\begin{array}{c}61 \\
(51-66)\end{array}$ & $92(71.9 \%)$ & $\begin{array}{c}92 \\
(71.9 \%)\end{array}$ & $\begin{array}{c}49 \\
(38.3 \%)\end{array}$ & $67(52.3 \%)$ & $2(1.6 \%)$ & $13(10.2 \%)$ & $\begin{array}{c}98 \\
(76.6 \%)\end{array}$ \\
\hline & & $p$ value & 0.708 & 1 & 0.226 & 0.112 & 0.211 & 1 & 0.072 & 0.652 \\
\hline
\end{tabular}

CHD: coronary heart disease; IFG: impaired fasting glucose; NG: normal glucose; PSM: propensity score match.

and more severe degrees of CHD when FPG levels were $>5.2 \mathrm{mmol} / \mathrm{L}$.

Our results showed several high ORs, especially when the cut-off point was $6.1 \mathrm{mmol} / \mathrm{L}$ in men, wherein the OR was 8.80 . The possible reason was that, compared with prospective analyses, all patients in this study received coronary angiography, which excludes the effects of false negatives. In prospective cohort studies, there may be patients who actually had CHD but did not visit the hospital for examination, such as in cases of asymptomatic myocardial ischemia. The presence of false negatives may also lead to an underestimation of blood glucose effects on $\mathrm{CHD}$, which was the reason why every patient we selected in this study had to have undergone coronary angiography.
The cut-off point for IFG was redefined in this study, with the cut-off of $5.2 \mathrm{mmol} / \mathrm{L}$ lower than that reported by the WHO and ADA. A study in Canada found that even glycemia under the diagnostic criteria of gestational diabetes can increase the risk of cardiovascular disease after several years of exposure [15]. Abnormal glucose metabolism damages blood vessels earlier than originally expected. In addition to the direct damage of vascular endothelial function caused by hyperglycemia, the insulin resistance and hyperinsulinemia already present would promote the formation of atherosclerosis even before the rise in blood glucose levels [16]. However, it may be difficult to maintain fasting glucose levels below $5.2 \mathrm{mmol} / \mathrm{L}$ using drugs alone. Excessive drug application may increase the risk of hypoglycemia, which also causes vascular damage [17]. We can, 


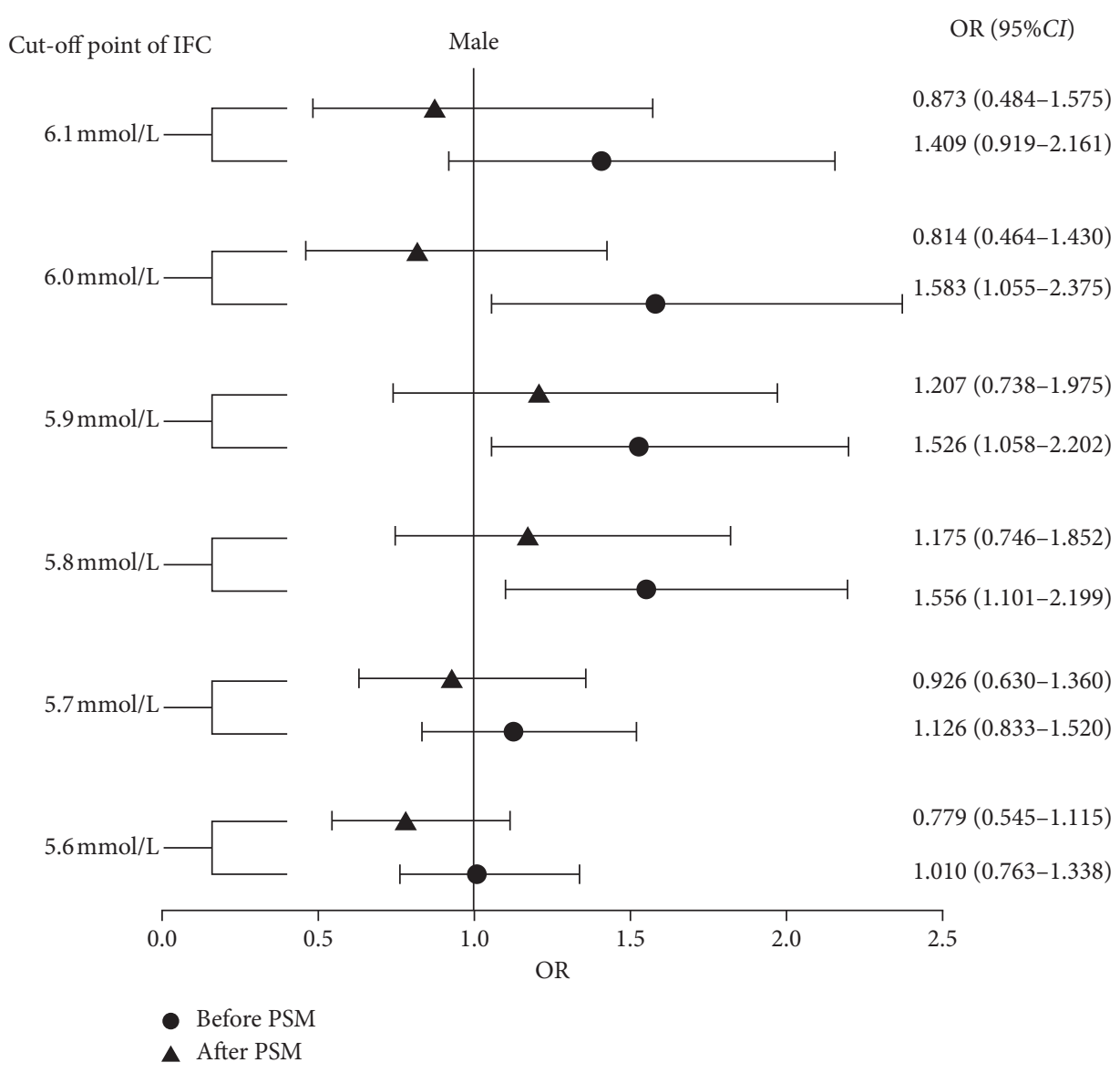

FIGURE 1: Odds ratio of IFG defined by the different cut-off points in males to the incidence of CHD. CHD: coronary heart disease; CI: confidence interval; IFG: impaired fasting glucose; OR: odds ratio; PSM: propensity score match.

TABLE 4: Baseline characteristics of IFG and normal groups defined by different cut-off points before and after PSM in female patients.

\begin{tabular}{|c|c|c|c|c|c|c|c|c|c|c|}
\hline \multicolumn{3}{|c|}{ Cut-off point of IFG } & Age & Hypertension & Smoking & Drinking & Dyslipidemia & $\begin{array}{c}\text { Chronic } \\
\text { kidney } \\
\text { disease }\end{array}$ & $\begin{array}{c}\text { Previous } \\
\text { ischemic } \\
\text { stroke }\end{array}$ & CHD \\
\hline \multirow{5}{*}{$5 \mathrm{mmol} / \mathrm{L}$} & \multirow{3}{*}{$\begin{array}{l}\text { Before } \\
\text { PSM }\end{array}$} & $\begin{array}{c}\text { NG } \\
(n=387)\end{array}$ & $\begin{array}{c}56 \\
(50-65)\end{array}$ & $262(67.7 \%)$ & $3(0.8 \%)$ & $3(0.8 \%)$ & $128(33.1 \%)$ & $3(0.8 \%)$ & $37(9.6 \%)$ & $\begin{array}{c}177 \\
(45.7 \%)\end{array}$ \\
\hline & & $\begin{array}{c}\text { IFG } \\
(n=639)\end{array}$ & $\begin{array}{c}61 \\
(53-66)\end{array}$ & $461(72.1 \%)$ & $9(1.4 \%)$ & $6(0.9 \%)$ & $236(36.9 \%)$ & $4(0.6 \%)$ & $49(7.7 \%)$ & $\begin{array}{c}360 \\
(56.3 \%)\end{array}$ \\
\hline & & $p$ value & $<0.001$ & 0.130 & 0.551 & 1 & 0.211 & 1 & 0.289 & 0.001 \\
\hline & \multirow{3}{*}{$\begin{array}{l}\text { After } \\
\text { PSM }\end{array}$} & $\begin{array}{c}\text { NG } \\
(n=376)\end{array}$ & $\begin{array}{c}57 \\
(51-65)\end{array}$ & $252(67 \%)$ & $3(0.8 \%)$ & $3(0.8 \%)$ & $126(33.5 \%)$ & $3(0.8 \%)$ & $34(9 \%)$ & $\begin{array}{c}167 \\
(44.4 \%)\end{array}$ \\
\hline & & $\begin{array}{c}\text { IFG } \\
(n=376)\end{array}$ & $\begin{array}{c}57 \\
(52-65)\end{array}$ & $259(68.9 \%)$ & $2(0.5 \%)$ & $2(0.5 \%)$ & $146(38.8 \%)$ & $1(0.3 \%)$ & $34(9 \%)$ & $\begin{array}{c}193 \\
(51.3 \%)\end{array}$ \\
\hline \multirow{7}{*}{$5.1 \mathrm{mmol} / \mathrm{L}$} & & $p$ value & 0.755 & 0.584 & 1 & 1 & 0.129 & 0.624 & 1 & 0.058 \\
\hline & \multirow{3}{*}{$\begin{array}{c}\text { Before } \\
\text { PSM }\end{array}$} & $\begin{array}{c}\text { NG } \\
(n=462)\end{array}$ & $\begin{array}{c}57 \\
(50 \sim 65)\end{array}$ & $309(66.9 \%)$ & $3(0.6 \%)$ & $3(0.6 \%)$ & $152(32.9 \%)$ & $3(0.6 \%)$ & $41(8.9 \%)$ & $\begin{array}{c}214 \\
(46.3 \%)\end{array}$ \\
\hline & & $\begin{array}{c}\text { IFG } \\
(n=564)\end{array}$ & $\begin{array}{c}61 \\
(53-67)\end{array}$ & $414(73.4 \%)$ & $9(1.6 \%)$ & $6(1.1 \%)$ & $212(37.6 \%)$ & $4(0.7 \%)$ & $45(8.0 \%)$ & $\begin{array}{c}323 \\
(57.3 \%)\end{array}$ \\
\hline & & $p$ value & $<0.001$ & 0.023 & 0.161 & 0.525 & 0.118 & 1 & 0.606 & $<0.001$ \\
\hline & \multirow{3}{*}{$\begin{array}{l}\text { After } \\
\text { PSM }\end{array}$} & $\begin{array}{c}\text { NG } \\
(n=400)\end{array}$ & $\begin{array}{c}60 \\
(52-65)\end{array}$ & $289(72.3 \%)$ & $1(0.3 \%)$ & $2(0.5 \%)$ & $133(33.3 \%)$ & $3(0.8 \%)$ & $41(10.3 \%)$ & $\begin{array}{c}179 \\
(44.8 \%)\end{array}$ \\
\hline & & $\begin{array}{c}\text { IFG } \\
(n=400)\end{array}$ & $\begin{array}{c}60 \\
(53-65)\end{array}$ & $264(66.0 \%)$ & $5(1.3 \%)$ & $5(1.3 \%)$ & $141(35.3 \%)$ & $3(0.8 \%)$ & $29(7.3 \%)$ & $\begin{array}{c}203 \\
(50.8 \%)\end{array}$ \\
\hline & & $p$ value & 0.523 & 0.056 & 0.217 & 0.255 & 0.551 & 1 & 0.133 & 0.089 \\
\hline
\end{tabular}


TABLE 4: Continued.

\begin{tabular}{|c|c|c|c|c|c|c|c|c|c|c|}
\hline Cut-of & point $o$ & IFG & Age & Hypertension & Smoking & Drinking & Dyslipidemia & $\begin{array}{c}\text { Chronic } \\
\text { kidney } \\
\text { disease }\end{array}$ & $\begin{array}{c}\text { Previous } \\
\text { ischemic } \\
\text { stroke }\end{array}$ & CHD \\
\hline \multirow{5}{*}{$5.2 \mathrm{mmol} / \mathrm{L}$} & \multirow{3}{*}{$\begin{array}{l}\text { Before } \\
\text { PSM }\end{array}$} & $\begin{array}{c}\mathrm{NG} \\
(n=528)\end{array}$ & $\begin{array}{c}58 \\
(51-65)\end{array}$ & 357 (67.6\%) & $6(1.1 \%)$ & $4(0.8 \%)$ & $181(34.3 \%)$ & $3(0.6 \%)$ & $42(8.0 \%)$ & $\begin{array}{c}250 \\
(47.3 \%)\end{array}$ \\
\hline & & $\begin{array}{c}\text { IFG } \\
(n=498)\end{array}$ & $\begin{array}{c}61 \\
(53-67)\end{array}$ & $366(73.5 \%)$ & $6(1.2 \%)$ & $5(1.0 \%)$ & $183(36.7 \%)$ & $4(0.8 \%)$ & 44 (8.8\%) & $\begin{array}{c}287 \\
(57.6 \%)\end{array}$ \\
\hline & & $p$ value & $<0.001$ & 0.039 & 0.919 & 0.747 & 0.409 & 0.718 & 0.611 & 0.001 \\
\hline & \multirow[b]{2}{*}{$\begin{array}{l}\text { After } \\
\text { PSM }\end{array}$} & $\begin{array}{c}\text { NG } \\
(n=431)\end{array}$ & $\begin{array}{c}60 \\
(53-66)\end{array}$ & $318(73.8 \%)$ & $4(0.9 \%)$ & $2(0.5 \%)$ & $145(33.6 \%)$ & $3(0.7 \%)$ & $40(9.3 \%)$ & $\begin{array}{c}194 \\
(45.0 \%)\end{array}$ \\
\hline & & $\begin{array}{c}\text { IFG } \\
(n=431)\end{array}$ & $\begin{array}{c}60 \\
(53-65)\end{array}$ & $305(70.8 \%)$ & $5(1.2 \%)$ & $5(1.2 \%)$ & $153(35.5 \%)$ & $4(0.9 \%)$ & 37 (8.6\%) & $\begin{array}{c}233 \\
(54.1 \%)\end{array}$ \\
\hline \multirow{7}{*}{$5.3 \mathrm{mmol} / \mathrm{L}$} & \multirow{4}{*}{$\begin{array}{l}\text { Before } \\
\text { PSM }\end{array}$} & $p$ value & 0.709 & 0.323 & 1 & 0.451 & 0.567 & 1 & 0.720 & 0.008 \\
\hline & & $\begin{array}{c}\text { NG } \\
(n=595)\end{array}$ & $\begin{array}{c}58 \\
(51-65)\end{array}$ & $406(68.2 \%)$ & $7(1.2 \%)$ & $5(0.8 \%)$ & $204(34.3 \%)$ & $3(0.5 \%)$ & 49 (8.2\%) & $\begin{array}{c}290 \\
(48.7 \%)\end{array}$ \\
\hline & & $\begin{array}{c}\text { IFG } \\
(n=431)\end{array}$ & $\begin{array}{c}61 \\
(53-67)\end{array}$ & $317(73.5 \%)$ & $5(1.2 \%)$ & $4(0.9 \%)$ & $160(37.1 \%)$ & $4(0.9 \%)$ & 37 (8.6\%) & $\begin{array}{c}247 \\
(57.3 \%)\end{array}$ \\
\hline & & $p$ value & $<0.001$ & 0.065 & 0.981 & 1 & 0.348 & 0.462 & 0.842 & 0.007 \\
\hline & \multirow{3}{*}{$\begin{array}{l}\text { After } \\
\text { PSM }\end{array}$} & $\begin{array}{c}\text { NG } \\
(n=414)\end{array}$ & $60 \pm 9$ & $285(68.8 \%)$ & $3(0.7 \%)$ & $2(0.5 \%)$ & $138(33.3 \%)$ & $3(0.7 \%)$ & $38(9.2 \%)$ & $\begin{array}{c}160 \\
(38.6 \%)\end{array}$ \\
\hline & & $\begin{array}{c}\text { IFG } \\
(n=414)\end{array}$ & $60 \pm 9$ & $303(73.2 \%)$ & $5(1.2 \%)$ & $4(1.0 \%)$ & $153(37.0 \%)$ & $4(1.0 \%)$ & 35 (8.5\%) & $\begin{array}{c}231 \\
(55.8 \%)\end{array}$ \\
\hline & & $p$ value & 0.981 & 0.168 & 0.725 & 0.686 & 0.275 & 1 & 0.713 & $<0.001$ \\
\hline \multirow{5}{*}{$5.4 \mathrm{mmol} / \mathrm{L}$} & \multirow{3}{*}{$\begin{array}{l}\text { Before } \\
\text { PSM }\end{array}$} & $\begin{array}{c}\text { NG } \\
(n=640)\end{array}$ & $\begin{array}{c}58 \\
(52-65)\end{array}$ & $439(68.6 \%)$ & $8(1.3 \%)$ & $5(0.8 \%)$ & $215(33.6 \%)$ & $3(0.5 \%)$ & $53(8.3 \%)$ & $\begin{array}{c}316 \\
(49.4 \%)\end{array}$ \\
\hline & & $\begin{array}{c}\text { IFG } \\
(n=386)\end{array}$ & $\begin{array}{c}61 \\
(53-67)\end{array}$ & $284(73.6 \%)$ & $4(1.0 \%)$ & $4(1.0 \%)$ & $149(38.6 \%)$ & $4(1.0 \%)$ & $33(8.5 \%)$ & $\begin{array}{c}221 \\
(57.3 \%)\end{array}$ \\
\hline & & $p$ value & $<0.001$ & 0.090 & 1 & 0.735 & 0.104 & 0.436 & 0.881 & 0.014 \\
\hline & \multirow{3}{*}{$\begin{array}{l}\text { After } \\
\text { PSM }\end{array}$} & $\begin{array}{c}\text { NG } \\
(n=386)\end{array}$ & $\begin{array}{c}60 \\
(53-67)\end{array}$ & $282(73.1 \%)$ & $4(1.0 \%)$ & $2(0.5 \%)$ & $144(37.3 \%)$ & $3(0.8 \%)$ & $32(8.3 \%)$ & $\begin{array}{c}117 \\
(30.3 \%)\end{array}$ \\
\hline & & $\begin{array}{c}\text { IFG } \\
(n=386)\end{array}$ & $\begin{array}{c}61 \\
(53-67)\end{array}$ & $284(73.6 \%)$ & $4(1.0 \%)$ & $4(1.0 \%)$ & $149(38.6 \%)$ & $4(1.0 \%)$ & $33(8.5 \%)$ & $\begin{array}{c}221 \\
(57.3 \%)\end{array}$ \\
\hline \multirow{7}{*}{$5.5 \mathrm{mmol} / \mathrm{L}$} & & $p$ value & 0.204 & 0.871 & 1 & 0.686 & 0.711 & 1 & 0.897 & $<0.001$ \\
\hline & \multirow{3}{*}{$\begin{array}{l}\text { Before } \\
\text { PSM }\end{array}$} & $\begin{array}{c}\text { NG } \\
(n=688)\end{array}$ & $\begin{array}{c}59 \\
(52-65)\end{array}$ & 467 (67.9\%) & $8(1.2 \%)$ & $5(0.7 \%)$ & $236(34.3 \%)$ & $3(0.4 \%)$ & $53(7.7 \%)$ & $\begin{array}{c}341 \\
(49.6 \%)\end{array}$ \\
\hline & & $\begin{array}{c}\text { IFG } \\
(n=338)\end{array}$ & $\begin{array}{c}61 \\
(54-68)\end{array}$ & $256(75.7 \%)$ & $4(1.2 \%)$ & $4(1.2 \%)$ & $128(37.9 \%)$ & $4(1.2 \%)$ & $33(9.8 \%)$ & $\begin{array}{c}196 \\
(58.0 \%)\end{array}$ \\
\hline & & $p$ value & $<0.001$ & 0.009 & 1 & 0.487 & 0.262 & 0.227 & 0.263 & 0.011 \\
\hline & \multirow{3}{*}{$\begin{array}{l}\text { After } \\
\text { PSM }\end{array}$} & $\begin{array}{c}\text { NG } \\
(n=336)\end{array}$ & $\begin{array}{c}61 \\
(53-67)\end{array}$ & $264(78.6 \%)$ & $4(1.2 \%)$ & $2(0.6 \%)$ & $105(31.3 \%)$ & $3(0.9 \%)$ & $33(9.8 \%)$ & $\begin{array}{c}87 \\
(25.9 \%)\end{array}$ \\
\hline & & $\begin{array}{c}\text { IFG } \\
(n=336)\end{array}$ & $\begin{array}{c}61 \\
(54-68)\end{array}$ & $254(75.6 \%)$ & $4(1.2 \%)$ & $4(1.2 \%)$ & $127(37.8 \%)$ & $4(1.2 \%)$ & $32(9.5 \%)$ & $\begin{array}{c}194 \\
(57.7 \%)\end{array}$ \\
\hline & & $p$ value & 0.660 & 0.359 & 1 & 0.686 & 0.074 & 1 & 0.896 & $<0.001$ \\
\hline \multirow{5}{*}{$5.6 \mathrm{mmol} / \mathrm{L}$} & \multirow{3}{*}{$\begin{array}{l}\text { Before } \\
\text { PSM }\end{array}$} & $\begin{array}{c}\text { NG } \\
(n=735)\end{array}$ & $\begin{array}{c}59 \\
(52-65)\end{array}$ & 499 (67.9\%) & $8(1.1 \%)$ & $6(0.8 \%)$ & $255(34.7 \%)$ & $3(0.4 \%)$ & $56(7.6 \%)$ & $\begin{array}{c}362 \\
(49.3 \%)\end{array}$ \\
\hline & & $\begin{array}{c}\text { IFG } \\
(n=291)\end{array}$ & $\begin{array}{c}61 \\
(54-68)\end{array}$ & $224(77.0 \%)$ & $4(1.4 \%)$ & $3(1.0 \%)$ & $109(37.5 \%)$ & $4(1.4 \%)$ & $30(10.3 \%)$ & $\begin{array}{c}175 \\
(60.1 \%)\end{array}$ \\
\hline & & $p$ value & $<0.001$ & 0.004 & 0.749 & 0.719 & 0.404 & 0.105 & 0.161 & 0.002 \\
\hline & \multirow{3}{*}{$\begin{array}{l}\text { After } \\
\text { PSM }\end{array}$} & $\begin{array}{c}\text { NG } \\
(n=291)\end{array}$ & $\begin{array}{c}61 \\
(53-67)\end{array}$ & $234(80.4 \%)$ & $4(1.4 \%)$ & $2(0.7 \%)$ & $100(34.4 \%)$ & $2(0.7 \%)$ & $27(9.3 \%)$ & $\begin{array}{c}59 \\
(20.3 \%)\end{array}$ \\
\hline & & $\begin{array}{c}\text { IFG } \\
(n=291)\end{array}$ & $\begin{array}{c}61 \\
(54-68)\end{array}$ & $224(77.0 \%)$ & $4(1.4 \%)$ & $3(1.0 \%)$ & $109(37.5 \%)$ & $4(1.4 \%)$ & $30(10.3 \%)$ & $\begin{array}{c}175 \\
(60.1 \%)\end{array}$ \\
\hline \multirow{7}{*}{$6.1 \mathrm{mmol} / \mathrm{L}$} & & $p$ value & 0.531 & 0.311 & 1 & 1 & 0.437 & 0.686 & 0.781 & $<0.001$ \\
\hline & \multirow{3}{*}{$\begin{array}{l}\text { Before } \\
\text { PSM }\end{array}$} & $\begin{array}{c}\text { NG } \\
(n=894)\end{array}$ & $\begin{array}{c}59 \\
(52-65)\end{array}$ & $620(69.4 \%)$ & $9(1.0 \%)$ & $7(0.8 \%)$ & $313(35.0 \%)$ & $7(0.8 \%)$ & $71(7.9 \%)$ & $\begin{array}{c}456 \\
(51.0 \%)\end{array}$ \\
\hline & & $\begin{array}{c}\text { IFG } \\
(n=132)\end{array}$ & $\begin{array}{c}62 \\
(55-68)\end{array}$ & $103(78.0 \%)$ & $3(2.3 \%)$ & $2(1.5 \%)$ & $51(38.6 \%)$ & $0(0.0 \%)$ & 15 (11.4\%) & $\begin{array}{c}81 \\
(61.4 \%)\end{array}$ \\
\hline & & $p$ value & 0.002 & 0.041 & 0.193 & 0.326 & 0.416 & 0.604 & 0.185 & 0.026 \\
\hline & \multirow{3}{*}{$\begin{array}{l}\text { After } \\
\text { PSM }\end{array}$} & $\begin{array}{c}\text { NG } \\
(n=130)\end{array}$ & $61 \pm 9$ & $103(79.2 \%)$ & $2(1.5 \%)$ & $0(0 \%)$ & $51(39.2 \%)$ & $1(0.8 \%)$ & $9(6.9 \%)$ & $\begin{array}{c}20 \\
(15.4 \%)\end{array}$ \\
\hline & & $\begin{array}{c}\text { IFG } \\
(n=130)\end{array}$ & $61 \pm 9$ & $101(77.7 \%)$ & $3(2.3 \%)$ & $2(1.5 \%)$ & $51(39.2 \%)$ & $0(0.0 \%)$ & $14(10.8 \%)$ & $\begin{array}{c}80 \\
(61.5 \%)\end{array}$ \\
\hline & & $p$ value & 0.861 & 0.763 & 1 & 0.498 & 1 & 1 & 0.275 & $<0.001$ \\
\hline
\end{tabular}




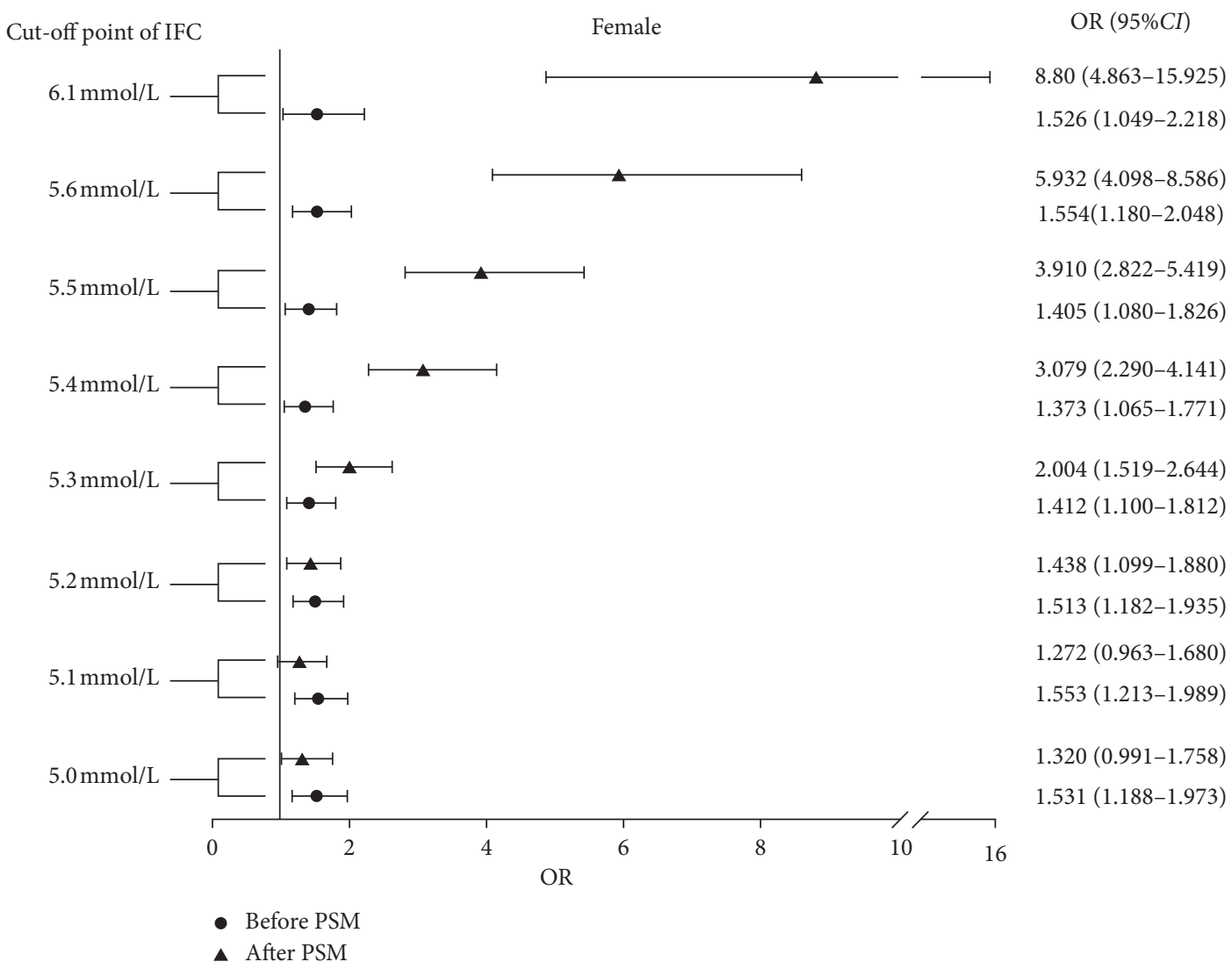

Figure 2: Odds ratio of IFG defined by the different cut-off points in females to the incidence of CHD. CHD: coronary heart disease; CI: confidence interval; IFG: impaired fasting glucose; OR: odds ratio; PSM: propensity score match.

TABLE 5: Relationship between IFG defined by $5.2 \mathrm{mmol} / \mathrm{L}$ and degree of lesion.

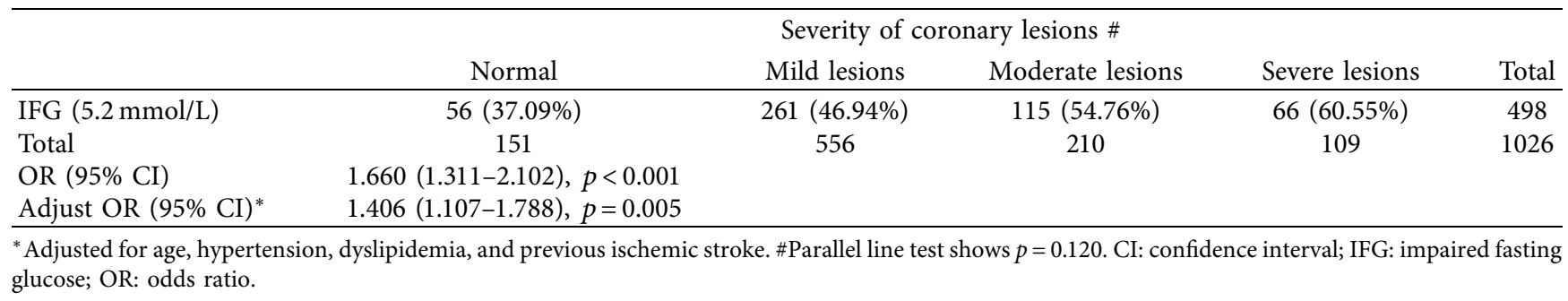

TABLE 6: Differences in baseline characteristics between sexes in patients with coronary heart disease.

\begin{tabular}{lcccccccc}
\hline & Age & Hypertension & Dyslipidemia & Smoking & Drinking & $\begin{array}{c}\text { Previous ischemic } \\
\text { stroke }\end{array}$ & $\begin{array}{c}\text { Chronic kidney } \\
\text { disease }\end{array}$ & FPG \\
\hline $\begin{array}{l}\text { Male } \\
(n=914)\end{array}$ & $59(51-66)$ & $599(65.5 \%)$ & $479(52.4 \%)$ & $612(67.0 \%)$ & $306(33.5 \%)$ & $113(12.4 \%)$ & $15(1.6 \%)$ & $5.07(4.68-5.56)$ \\
$\begin{array}{l}\text { Female } \\
(n=537)\end{array}$ & $62(54-68)$ & $422(78.6 \%)$ & $215(40.0 \%)$ & $9(1.7 \%)$ & $6(1.1 \%)$ & $55(10.2 \%)$ & $3(0.6 \%)$ & $5.25(4.86-5.74)$ \\
$p$ & $<0.001$ & $<0.001$ & $<0.001$ & $<0.001$ & $<0.001$ & 0.223 & 0.072 & $<0.001$ \\
\hline
\end{tabular}

FPG: fasting plasma glucose.

however, improve the body's metabolic state and insulin sensitivity by adjusting lifestyles, increasing exercise, and reducing weight, thereby reducing the risk of CHD [18-20].
It is worth mentioning that the definition of "impaired fasting glucose" in this study only accounted for the incidence and degree of CHD, while the concept of IFG is in fact 


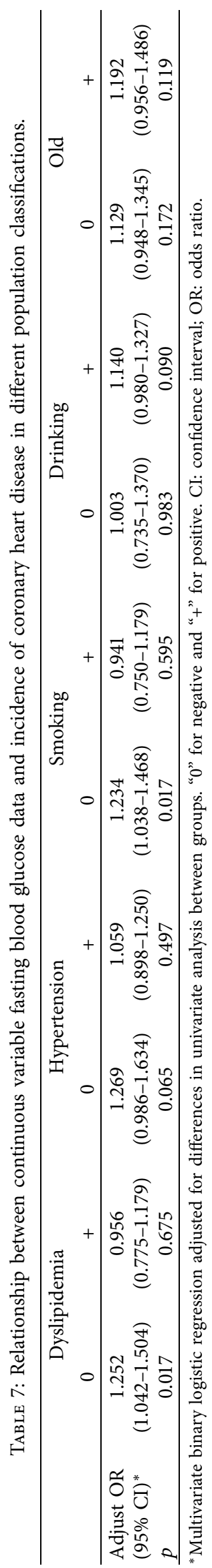


more complex; for example, whether IFG will develop into clinically diagnosed diabetes in the next few years or whether IFG causes an increase in the incidence of other cardiovascular diseases remains unclear.

4.2. Comparison of Risk Factors between Men and Women. Similar to the Framingham cohort study, we found discrepancies in the results between men and women. Elevated FPG levels in men were not associated with an increase in CHD incidence. This may be due to the inherent differences between sexes, such as the differences in chromosomes or in estrogen levels. It may also be the differences in composition of risk factor profiles. In comparison with patients having positive angiographic results in both men and women, male CHD patients had lower FPG levels, age of onset, and rate of hypertension, but higher rates of smoking, drinking, and dyslipidemia; all 3 factors damage the cardiovascular system. Further analysis showed that dyslipidemia and smoking in the population did not affect the incidence of CHD, whereas the opposite was observed in those without dyslipidemia and who were nonsmokers. This was likely due to the higher dyslipidemia and smoking rates in men, which could have masked the damage to blood vessels, caused by elevated FPG levels, and thus may have obscured the effects of IFG on $\mathrm{CHD}$ incidence in men. This can partly explain the difference between men and women regarding the impact of IFG on CHD incidence. In rural China, men constitute the primary labor force and are burdened with more physical tasks. Physical activity can improve vascular endothelial function, reduce body inflammatory response and visceral fat, and provide other benefits, as well as limit the incidence of CHD. This may also be the reason why IFG did not affect the incidence of CHD in men [21].

4.3. Study Limitations. This project was single-centered, and the selected population was exclusively from the northern region of Henan Province. Thus, the results obtained likely do not represent all rural areas of China. However, there are more than 40 million people living in the rural areas of Henan Province; thus, our research provides critical insight into the health of these individuals.

The Clinical Data and Sample Resource Research Center of Cardiovascular Diseases in the Henan Province has only recently been established, and the available sample size was therefore limited, with existing data consisting mostly of individuals from a rural population. Therefore, it was difficult to compare and analyze the differences between urban and rural populations. However, the database is continuously updated, and more information will be available for future research.

Furthermore, several important data like body mass index and CHD family history were not available in the current database, which impacted the results of our research. The present investigation also did not intend to use FPG as the assessment of diabetes status. There may have been individuals with normal FPG levels but with 2-hour postload glucose levels that fit the diagnostic criteria for diabetes.

\section{Conclusion}

The incidence of CHD increased in diabetes-free women in rural areas of northern Henan when the FPG level was greater than $5.2 \mathrm{mmol} / \mathrm{L}$. It is hence recommended that women in this region be more active in managing their blood glucose levels and should adopt healthy lifestyle interventions as early as possible, especially individuals with other risk factors. Although the incidence of CHD in men was not affected by the increase in FPG, risk factors, such as smoking, should be limited to control blood lipid levels.

\section{Data Availability}

The data used to support the findings of this study are available from the corresponding author upon request.

\section{Conflicts of Interest}

The authors declare no conflicts of interest regarding the publication of this paper.

\section{Authors' Contributions}

Quan Guo and Fei Lin contributed equally to this work and should be considered as the co-first authors.

\section{Acknowledgments}

The authors would like to acknowledge Editage (https:// www.editage.cn) for English language editing. The study was funded by the Key Scientific Research Projects of Higher Education Institutions in Henan Province (no. 18A320005 and no. 19A360032) and Scientific and Technological Research Projects in Henan Province (no. 182102310182).

\section{References}

[1] T. R. Einarson, A. Acs, C. Ludwig, and U. H. Panton, "Prevalence of cardiovascular disease in type 2 diabetes: a systematic literature review of scientific evidence from across the world in 2007-2017," Cardiovascular Diabetology, vol. 17, p. $83,2018$.

[2] M. Buysschaert, J. L. Medina, M. Bergman, A. Shah, and J. Lonier, "Prediabetes and associated disorders," Endocrine, vol. 48, no. 2, pp. 371-393, 2015.

[3] C. H. Chou, W. C. Tsai, M. C. Wang et al., "Effects of deranged glucose homeostasis on peripheral arterial stiffness index in patients with pre-diabetes mellitus," International Heart Journal, vol. 54, pp. 27-32, 2013.

[4] ADA, "Classification and diagnosis of diabetes: standards of medical care in diabetes-2019," Diabetes Care, vol. 42, pp. S13-S28, 2019.

[5] H.-K. Kim, C.-H. Kim, E. H. Kim et al., "Impaired fasting glucose and risk of cardiovascular disease in Korean men and women: the Korean Heart Study," Diabetes Care, vol. 36, no. 2, pp. 328-335, 2013.

[6] E. S. Ford, G. Zhao, and C. Li, "Pre-diabetes and the risk for cardiovascular disease," Journal of the American College of Cardiology, vol. 55, no. 13, pp. 1310-1317, 2010. 
[7] T. Xu, W. Liu, X. Cai et al., "Risk of coronary heart disease in different criterion of impaired fasting glucose: a meta-analysis," Medicine, vol. 94, p. e1740, 2015.

[8] Y. S. Levitzky, M. J. Pencina, R. B. D’Agostino et al., "Impact of impaired fasting glucose on cardiovascular disease," Journal of the American College of Cardiology, vol. 51, no. 3, pp. 264-270, 2008.

[9] C. M. Lawes, V. Parag, D. A. Bennett et al., "Blood glucose and risk of cardiovascular disease in the Asia Pacific region," Diabetes Care, vol. 27, no. 12, pp. 2836-2842, 2004.

[10] N. Sarwar and P. Gao, "Diabetes mellitus, fasting blood glucose concentration, and risk of vascular disease: A collaborative meta-analysis of 102 prospective studies," Lancet, vol. 375, pp. 2215-2222, 2010.

[11] B. B. Zhang, G. A. Zhao, M. Yang et al., "Birth month associates with risk of coronary artery disease and its complications: A propensity score matched analysis," Medicina clinica, vol. 153, no. 12, pp. 454-545, 2019.

[12] S. Yusuf, S. Rangarajan, K. Teo et al., "Cardiovascular risk and events in 17 low-, middle-, and high-income countries," New England Journal of Medicine, vol. 371, no. 9, pp. 818-827, 2014.

[13] D. Zhao, J. Liu, M. Wang, X. Zhang, and M. Zhou, "Epidemiology of cardiovascular disease in China: current features and implications," Nature Reviews Cardiology, vol. 16, no. 4, pp. 203-212, 2018.

[14] C. Sinning, L. Lillpopp, S. Appelbaum et al., "Angiographic score assessment improves cardiovascular risk prediction: the clinical value of SYNTAX and Gensini application," Clinical Research in Cardiology, vol. 102, no. 7, pp. 495-503, 2013.

[15] R. Retnakaran and B. R. Shah, "Glucose screening in pregnancy and future risk of cardiovascular disease in women: a retrospective, population-based cohort study," The Lancet Diabetes \& Endocrinology, vol. 7, no. 5, pp. 378-384, 2019.

[16] S. J. Bigornia, M. G. Farb, S. Tiwari et al., "Insulin status and vascular responses to weight loss in obesity," Journal of the American College of Cardiology, vol. 62, no. 24, pp. 2297-2305, 2013.

[17] M. Murata, H. Adachi, and S. Oshima, "Asymptomatic reactive hypoglycemia and inflammatory reaction in patients with coronary artery disease," International Heart Journal, vol. 59, pp. 705-712, 2018.

[18] E. Ferrannini, "Definition of intervention points in prediabetes," The Lancet Diabetes \& Endocrinology, vol. 2, no. 8, pp. 667-675, 2014.

[19] G. Li, P. Zhang, J. Wang et al., "Cardiovascular mortality, allcause mortality, and diabetes incidence after lifestyle intervention for people with impaired glucose tolerance in the $\mathrm{Da}$ Qing Diabetes Prevention Study: a 23-year follow-up study," The Lancet Diabetes \& Endocrinology, vol. 2, no. 6, pp. 474480, 2014.

[20] L. A. Shin-Yu, T. Jia-Ling, L. Jian-Tao et al., "A systematic review and meta-analysis of the effect of lifestyle modification on metabolic control in overweight children," Evidence Based Complementary \& Alternative Medicine, vol. 2017, Article ID 5681909, 12 pages, 2017.

[21] L. Soares-Miranda, D. S. Siscovick, B. M. Psaty, W. T. Longstreth Jr, and D. Mozaffarian, "Physical activity and risk of coronary heart disease and stroke in older adults," Circulation, vol. 133, no. 2, pp. 147-155, 2016. 